\title{
SEMANTIC FUSION AND JOINT ANALYSIS OF TERAHERTZ AND 3D VIDEO IMAGES BY THE MEANS OF OBJECT-ORIENTED LOGIC PROGRAMMING
}

Alexei A. Morozov, Olga S. Sushkova, Alexander F. Polupanov, Gennady K. Mansurov, Stanislav K. Paprotskiy, Alexander V. Yanushko, Nadezda G. Petrova, Alexander S. Bugaev

Kotelnikov Institute of Radioengineering and Electronics of RAS, http:/ /www.cplire.ru

11/7, Mokhovaya str., Moscow 125009, Russian Federation

Vyacheslav E. Antsiperov

Moscow Institute of Physics and Technology, http://mipt.ru

9, Institutsky per., Dolgoprudny 141701, Moscow Region, Russian Federation

morozov@cplire.ru, o.sushkova@mail.ru,sashap55@mail.ru, antciperov@gmail.com,gmansurov@mail. ru,s.paprotskiy@gmail.com,yanushko@gmail.com, petrova@cplire.ru,bugaev@cplire.ru

Abstract. The aim of the work is to create methods and software tools for the terahertz range intelligent video surveillance, that is, for automatic analysis of video images in the terahertz frequency range. Terahertz video surveillance provides unique prospects in the area of public safety; in particular, it allows you to remotely identify weapons and dangerous items hidden under clothing on the human body. However, such characteristics of terahertz video, as low resolution, low contrast, and low signal-to-noise ratio, lead to the need to develop new methods and approaches to automatic video analysis. To understand the terahertz video image, the operator of the industrial video surveillance system usually compares it with images in other frequency ranges (visible or infrared). The comparison of these images of different types helps the operator to interpret the colored and/ or one-color spots in the terahertz image in a proper way. In terms of automatic video analysis, this means that the context of the observed events and objects is taken into account, or in other words, a semantic fusion is implemented of the terahertz range video image with video images of other frequency ranges, e.g., near-infrared, visible, etc. The authors consider the semantic fusion of the video images as a critical component of the prospective terahertz intelligent video surveillance technology. A new method of the terahertz video surveillance based on the fusion of the terahertz video with $3 \mathrm{D}$ video is proposed. The means of the object-oriented logic programming developed for the semantic fusion of the terahertz and $3 \mathrm{D}$ video images are described. The developed method provides a real-time fusion of the terahertz video acquired using the THERZ-7A (Astrohn Technology Ltd) subterahertz scanning device (0.23-0.27 $\mathrm{THz}$ ) and $3 \mathrm{D}$ video data acquired using the Kinect 2 (Microsoft Inc) time-of-flight sensor. The method and software tools for the semantic fusion of the terahertz and 3D video images are developed.

Keywords: terahertz video surveillance; $3 \mathrm{D}$ video surveillance; intelligent visual surveillance; objectoriented logic programming; Actor Prolog

UDC 510.663; 519.68:007.5; 519.68:681.513.7; 681.3.06

Bibliography - 83 references

Received 27.08.2018

RENSIT, 2018, 10(2):329-340

DOI: $10.17725 /$ rensit.2018.10.329

\section{Content}

1. Introduction (329)

2. Combining the possibilities of THz ANd $3 \mathrm{D}$ VIDEO: THE HISTORY OF THE QUESTION (331)

3. Experimental Setup (333)

4. Calibration of the measuring system (333)
5. Experimental Software (335)

6. Conclusion (336)

References (337)

\section{INTRODUCTION}

Terahertz (submillimeter) radiation is electromagnetic radiation with a frequency in the 
range of about $300 \mathrm{GHz}$ to 3 Terahertz, i.e. a wavelength of 0.1 to 1 millimeter. The indicated limits of the frequency range are conditional, and many researchers use this term for electromagnetic radiation ranging from tens of gigahertz to 10 terahherts [1-4]. In addition, since terahertz radiation is located on the boundary between infrared radiation and millimeter waves, in many reviews and technical applications they are treated together [5-11]. The term "subterahertz radiation" is also used, if it is necessary to emphasize that we are talking about electromagnetic waves with a frequency of less than one terahertz.

The huge interest of researchers in terahertz radiation is due to the fact that it easily passes through most dielectrics, including plastic, ceramics, brick, wood, cardboard and fabric and is not ionizing. Moreover, terahertz radiation, as well as thermal radiation, is widespread in nature; living organisms are natural sources of terahertz radiation. These properties of terahertz radiation make its use in medicine very attractive [7, 12], archeology, art criticism [9] and, of course, security systems [10, 11, 13-24]. A passive receiver of terahertz waves emitted by a human body allows you to detect dangerous objects and weapons hidden under clothing. Also, active terahertz video surveillance systems including backlighting in the terahertz waveband are being developed [25-27].

Despite intensive research in the field of terahertz video surveillance, it is currently not widely used, with the exception of the security systems of certain buildings and airports. This is due not only to technical difficulties in the generation and reception of terahertz waves (which is reflected, among other things, in the price of the equipment being developed), but also because the equipment currently in use does not allow the full potential of terahertz video surveillance to be fully realized. The resulting terahertz video images are characterized by low resolution, low contrast, low signal-to-noise ratio and a low frame rate. Nevertheless, commercial screening systems have already been created that allow you to reveal remotely and unnoticeably the presence of objects hidden under his clothing.

A typical terahertz video image looks like a set of blurry spots, color or monochrome, depending on the data visualization method used. Simultaneously with the video image in the terahertz frequency range, the screening system displays the image in the visible or infrared frequency range familiar to humans [28, 29] (Fig. 1). The terahertz image can be subjected to additional processing in order to increase the contrast and detect abnormal areas [30 34]. This allows a specially trained operator to correctly interpret the terahertz video image and to identify the presence of objects hidden on the human body. This use of terahertz video surveillance, of course, is not optimal. In particular, it gave rise to the opinion that terahertz video surveillance is ineffective for screening passengers at the airport, because the employee managing the CCTV system is easier to use for manual screening of passengers. It is obvious that the mass use of terahertz video surveillance systems will become possible only with the appearance of sufficiently reliable methods of fully automatic analysis of terahertz video images.

The purpose of this work is to create methods and means of terahertz intelligent video surveillance, that is, automatic analysis of video images in the terahertz frequency range. As a basis for the realization of terahertz intellectual video surveillance, the authors consider the semantic merging (comparison) of a terahertz video image with video images obtained on the basis of other physical principles.

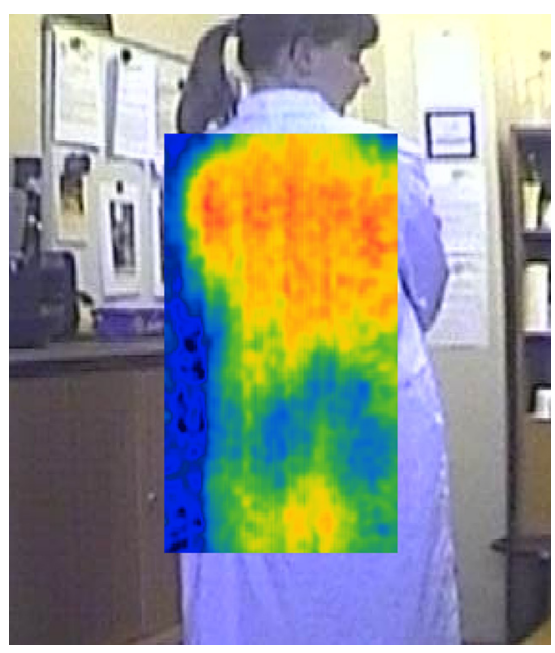

Fig. 1. An example of a terahertz video image. For the convenience of the video surveillance operator, a terahertz image is placed against the visible. The rainbow color scale was used to display the terabertz. data: red corresponds to the brightest areas of the image, blue to the coldest. The TT pistol bidden behind its back on a terahertz image looks like a blue-green spot. 
The article describes the experimental tools of object-oriented logical programming, developed at the Kotelnikov Institute of Radioengineering and Electronics of RAS for solving the problem of semantic fusion of terahertz and threedimensional video images. Section 2 provides a brief overview of existing projects aimed at combining the possibilities of terahertz and three-dimensional video. Section 3 describes the experimental setup used to test the methods for merging terahertz and three-dimensional video images. In Section 4, the problem of calibrating the measurement system, including the terahertz and time-of-flight cameras, is considered. Section 5 discusses the experimental software developed to implement the terahertz method of intelligent video surveillance, and an example of merging heterogeneous video data.

\section{COMBINING THE CAPABILITIES OF THZ AND 3D VIDEO: THE HISTORY OF THE ISSUE}

The idea of semantic fusion of video images is that data from different sources of video information are combined into one video image in such a way that the semantic content of some images affects the processing algorithms of others [35, 38]. The simplest example of the semantic fusion of video images in the terahertz and visible ranges can be considered the use of subtraction algorithms in the visible range to understand where a person is, and where the background image, and to distinguish (in different colors) objects found under human clothes and the background image Fig. 2).

Of course, the background subtraction algorithm can not distinguish the human body from other moving objects, however, for the operator of the inspection complex, the information obtained is sufficient, because it is able to understand exactly where the person is and what can correspond to those or other spots on the terahertz image. In the case of intelligent terahertz video surveillance for correct interpretation of the image of this information, of course, not enough. To exclude false positives of the system, it is necessary to take into account the data on the person's position in space, as well as on the position of individual parts of his body on the terahertz image. In this paper, we propose to use 3D vision tools for this purpose, namely, a depth map obtained with the Kinect 2 time-of-flight camera and skeletons calculated using the standard Kinekt 2 software.

The combination of three-dimensional machine vision with terahertz video surveillance is an area of intensive research and experimental development. It is customary to distinguish between passive and active terahertz video surveillance. Active terahertz video surveillance is historically associated with such areas as radar and holography [39, 41]. At present, in the field of active terahertz observation, it has been proposed to use the mechanical and digital beam formation [20, 42, 43], terahertz holography [44, 45], time-of-flight terahertz tomography [46], synthesized apertures [47] to obtain information on the threedimensional surface of the object under study -49], coding apertures [50-54], frequency modulation of the signal [55], antennas from metamaterials [56], etc. In the area of passive terahertz video surveillance, it was suggested to use the fusion of a terahertz image with 3D images, constructed using 3D-sensors based on structured light and lidar [57, 58], as well as data from multi-camera video [59]. Radar data merging with $3 \mathrm{D}$ and visible images is also used in active surveillance systems [60, 61].

At present, it is impossible to predict which terahertz video surveillance systems, active or passive, will find the most widespread. Both approaches have their advantages and disadvantages, and, in addition,
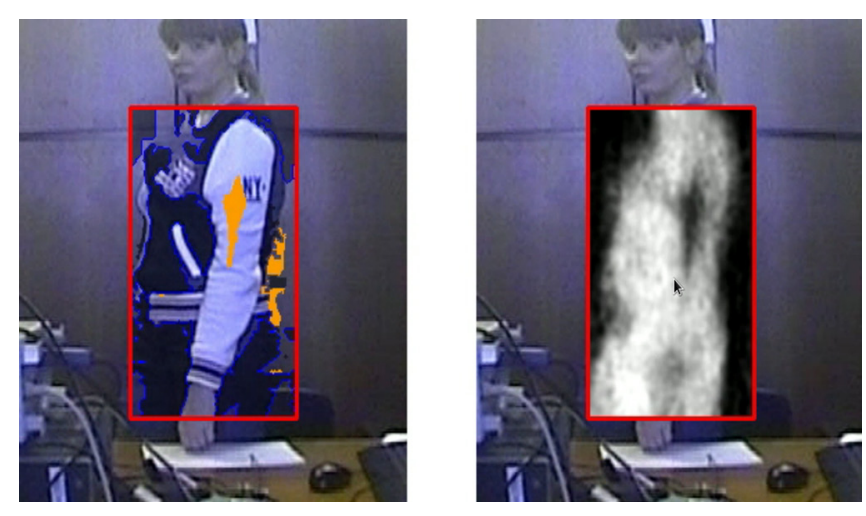

Fig. 2. An example of the visualization of terabertr data in the industrial inspection complex THERZ-7 A (Astrohn Technology $L t d)$. Software developed by ASoft. On the left is an image in the visible range. Orange marked areas on the human body, where, according to the assumption of the system, some objects are bidden. On the right is an image in the terahertz range, combined with a visible image. The position of the teraherts image relative to the visible image is indicated by a red frame. 
different ways of obtaining terahertz images give different information about the observed objects:

1. It is believed that active systems, theoretically, can provide a terahertz image of a higher quality, because they provide a better signal-to-noise ratio. However, one should take into account that passive systems accept and analyze their own radiation from people and objects (which can be useful, for example, for medical diagnostics); The hidden weapons and objects are clearly visible at the lumen against the background of the human body. Active systems take a reflected terahertz signal; the human body at the same time, as well as the objects hidden under the clothes, create glare that is difficult to interpret. In this situation, the problem of recognition is formulated quite differently.

2. Terahertz radiation, unlike $\mathrm{X}$-ray radiation, is not ionizing and therefore is considered to be safe for humans [62]. At the same time, the widespread use of active terahertz video surveillance systems will inevitably lead to increased electromagnetic pollution of the environment, and this will negatively affect people's health.

3. Active terahertz video surveillance system can be easily detected by electromagnetic radiation, therefore active video surveillance, in fact, ceases to be hidden. Passive systems, at the current level of development, also have drawbacks that prevent them from using for hidden video surveillance, for example, noise from the mechanical system of scanning terahertz radiation.

4. Studies in the field of active systems of terahertz 3D video surveillance are still at the level of experimental installations and prototypes. These systems are still quite cumbersome and inferior to passive terahertz video surveillance systems in terms of frame rate. Nevertheless, in the future such systems can provide information on the form of objects hidden under clothes, which will greatly facilitate their recognition.

5. Separate research areas are terahertz spectroscopy and its use for remote detection of weapons, drugs and explosives. Formally, terahertz spectroscopy systems can be attributed to active systems [16, 24, 63 -65], but they use terahertz radiation of a completely different frequency range. If it is advisable to use subterahertz waves located in the windows for the transparency of clothing $(0.1$ $\mathrm{THz}, 0.25 \mathrm{THz}$, etc.) for tanning clothes, then for terahertz spectroscopy it is necessary to use radiation corresponding to the absorption spectra of the investigated substances (0.5 THz and higher) [65].

To develop and test the method of fusion of terahertz and $3 \mathrm{D}$ video images, the industrial passive THERZ-7A THERZ-7A (manufactured by Astrohn Technology Ltd, operating frequency $0.23-0.27 \mathrm{THz}$ ) and the Time-of-Flight (ToF) camera of Kinekt 2 (Microsoft Inc). The developed method can also be adapted to the conditions of terahertz illumination. In the case of future industrial terahertz scanning systems scanning 3D surfaces in the terahertz range, the method will require some processing, since the existing algorithms for calculating skeletons of people are trained on video data in the near infrared and visible frequency bands.

\section{EXPERIMENTAL INSTALLATION}

To conduct experiments with terahertz video surveillance, an experimental setup was assembled, including the industrial THERZ-7A terahertz inspection complex and the Kinekt 2 device (Fig. 3). Both devices transmit not one, but several data streams at the same time. The passive terahertz complex transmits a series of terahertz video images with a resolution of $121 \times 207$ pixels and a frame rate of $6 \mathrm{~Hz}$ (in the proprietary format), as well as an image in the visible range with a resolution of $576 \times 704$ pixels and a frame rate of $25 \mathrm{~Hz}$ (in MJPEG format). The Kinect 2 device transmits a sequence of three-dimensional frames (coordinates of points in the $3 \mathrm{D}$ space) with a frame rate of 30 $\mathrm{Hz}$ and a matrix size of $512 \times 424$ pixels, a sequence of infrared images also at a frame rate of $30 \mathrm{~Hz}$ and a matrix size of $512 \times 424$ pixels, a skeleton sequence

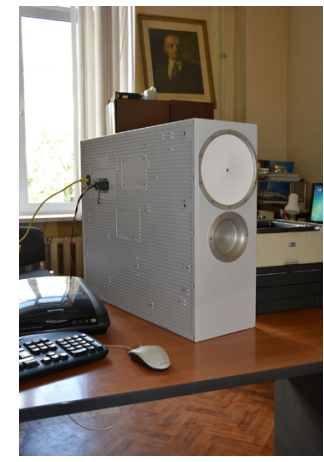

(a)

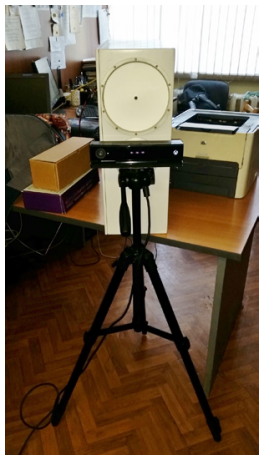

(b)

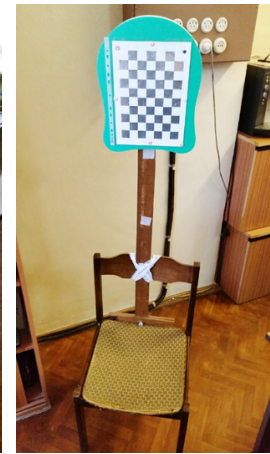

(c)
Fig. 3. An experimental setup that includes a passive terahert: receiver (a), a time-of-flight camera (b) and a calibration board (c). 
of people recognized in the frame 6 people at a time, each skeleton includes up to 25 nodes), a sequence of RGB-frames with a resolution of $1920 \times 1080$ pixels and a frame rate of $30 \mathrm{~Hz}$ and other information.

During data recording, the devices were connected to different computers, because the capacity (by recording) of the hard drive of one computer is not enough to record in real time the data issued by both devices. Note that the problem with the bandwidth of the hard disk is mainly due to the large volume of $3 \mathrm{D}$ data that the Kinekt 2 device provides. In conditions of practical use of the method of semantic fusion of video images, this problem can easily be solved by partial preprocessing and bringing the frequency of threedimensional frames to the frequency terahertz frames. Computer timers were synchronized before the experiments started, but this does not solve the problem of synchronization of video streams, and later the time shift between frames in different video streams still had to be checked and adjusted manually.

\section{CALIBRATION OF THE MEASURING SYSTEM}

Before each series of experiments, the experimental setup was calibrated. Calibration of the installation, including a terahertz detector and time-of-flight camera, is a separate technical task. When combining such devices, the same problems arise as when combining three-dimensional vision with a thermal imaging camera [66-68], namely, it becomes necessary to create a special calibration board whose elements will clearly differ in the images obtained with the help of both devices. An additional problem was that the terahertz image has an even lower resolution than what can be obtained with a typical thermal imaging camera. In this case, a small viewing angle of the terahertz camera does not allow to solve the problem by increasing the physical size of the calibration board.

To calibrate the experimental setup, the following approach was developed:

To determine the internal parameters of the optical system of the terahertz complex and its attachment to the time-of-flight camera, we took advantage of the fact that the terahertz image is rigidly tied to the image in the optical range given out

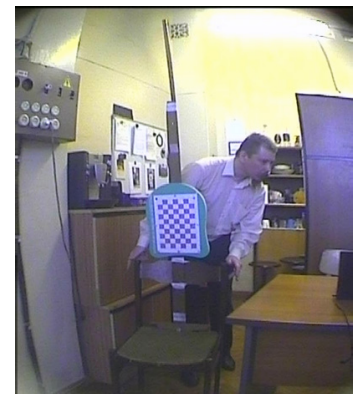

(a)

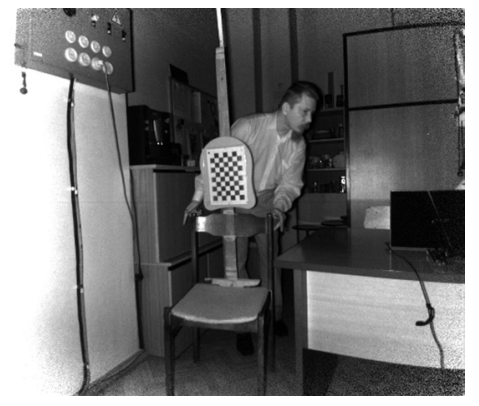

(b)
Fig. 4. The calibration process of the experimental setup. a) RGB image obtained from the terahertz complex. b) Infrared image obtained from the Kinelet 2 device. For the convenience of moving the calibration board along the crossbar, it is attached using strips of double-sided adhesive tape.

by the terahertz complex. For calibration, we used RGB images produced by the complex (Fig. 4a), and for experimental records - RGB images combined with terahertz ones (Fig. 1).

The Kinekt 2 device produces infrared images that appear as a by-product in the construction of depth maps (see Fig. 4b). This greatly simplifies the task of calibrating the time-of-flight camera, since there is no need to create a relief calibration board visible on the depth map.

For calibration, a standard chessboard pattern measuring $7 \times 10$ cells was used, the cell size was $25 \times 25 \mathrm{~mm}$. Dark cells of the board are made of aluminum foil, as this same calibration board is used for thermal imaging [69].

The calibration of the experimental setup includes the calculation of the internal parameters of the optical systems of each of the devices, and then the calculation of the mutual position of the devices and, on the basis of the information on the mutual position, further refinement of their internal parameters. For calculations, the Camera Calibration Toolbox for Matlab software was used [70], in particular, the stereopair calibration algorithm was used to calculate the mutual position of the cameras. The package [70] was developed more than 20 years ago, however, compared to the modern camera calibration package in the Matlab system, it is possible to calibrate a stereopair collected from two cameras with different matrix sizes. Calculation of internal and external parameters of the optical system is an optimization problem, therefore, in order to obtain a stable solution, it is necessary to correctly set limits on the space of values of the parameters 
of the model of the optical system. To calibrate the experimental setup, the following limitations were used: to permit the change of the position of the principal point, to take into account two coefficients of radial distortion (out of three possible ones), not to take into account the tangential distortion. To calibrate, up to 45 pairs of calibration images were used (Figure 4), this ensured the accuracy of fitting the model to approximately 0.18 pixels for Kinect 2 and 0.2 RGB pixels for the terahertz complex (Fig. 5).

Based on calibration data of the measuring setup, three-dimensional tables of correspondences between the point cloud recorded by the time-offlight camera and dots on the terahertz image were calculated. In the selected model of the measuring system, the three-dimensional correspondence table is a matrix of $512 \times 424$ pixels (the size of the time-offlight camera matrix) in which the lists of quadratic polynomial coefficients are written to calculate the coordinates on the RGB image produced by the terahertz complex. In each cell of the matrix $(i, j)$ six coefficients p1, p2, p3, q1, q2, q3 are written. The coordinates $(\mathrm{x}, \mathrm{y})$ in the RGB image are calculated using quadratic polynomials depending on the reciprocal distance $d(i, j)$ in meters from the timeof-flight camera to the point $(i, j)$ on the surface:

$$
\begin{aligned}
& \mathrm{x}=\mathrm{p} 1(1 / \mathrm{d}) 2+\mathrm{p} 2(1 / \mathrm{d})+\mathrm{p} 3, \\
& \mathrm{y}=\mathrm{q} 1(1 / \mathrm{d}) 2+\mathrm{q} 2(1 / \mathrm{d})+\mathrm{q} 3 .
\end{aligned}
$$

3D match tables are used for fast (in real time) fusion of video images received from different devices.

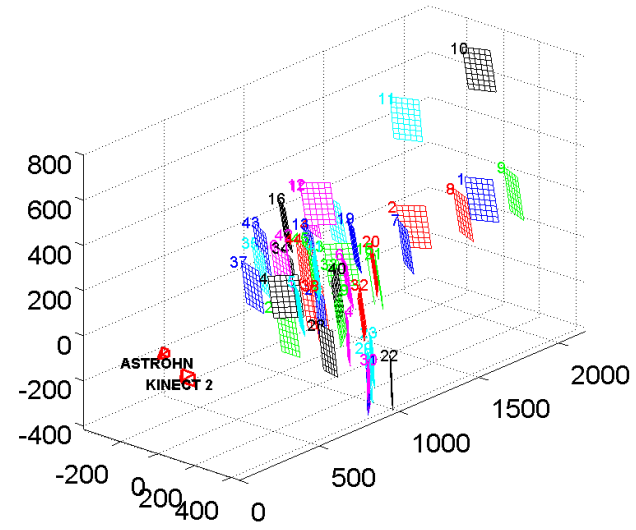

Fig. 5. The results of the joint calibration of terahertz and timeof-flight cameras as stereo pairs. For calculations, the software package Camera Calibration Toolbox for Matlab [70] was used. Dimensions on axes are in millimeters.

\section{EXPERIMENTAL SOFTWARE}

Working with three-dimensional correspondence tables is implemented in the built-in KinectBuffer class of the object-oriented logical language Actor Prolog [71-83]. This class is designed to receive data from the time-of-flight camera of the Kinect 2 device, and also to record and read the created $3 \mathrm{D}$ video. A detailed description of the built-in KinectBuffer class can be found in [69].

One of the functions of the built-in KinectBuffer class is the construction of a threedimensional surface based on a cloud of points and the projection onto this surface of a given texture using a previously prepared correspondence table. We used these tools to merge 3D and terahertz video images in our experiments. The main problem that arises in this way of merging video images is the synchronization of video streams with different frame rates. This problem is complicated by the fact that the frame rate in video streams is not constant. In particular, the frame rate of the skeleton stream produced by the standard SDK of the Kinekt 2 device may not correspond to the frequency of the 3D frames. To solve the problem of synchronization of video streams, a method of "advanced reading" of terahertz video data was developed. This method is implemented in a special built-in class of Actor Prologue Astrohn.

The built-in Astrohn class is designed to produce terahertz and RGB video data from the THERZ$7 \mathrm{~A}$ device. As well as the built-in KinectBuffer class, the built-in Astrohn class supports data input directly from an external device, as well as recording video data to a file and reading video data from a file. The Astrohn class supports the conversion of terahertz video data into color images. In particular, in the form of color images, terahertz data (using pseudocolours), RGB data transmitted by the terahertz complex, as well as RGB data combined with the current terahertz image can be presented directly. The Astrohn class supports the simplest mode of video frame synchronization, which combines terahertz and RGB images, obtained as close as possible in time. Currently, the Astrohn class supports more than 25 high resolution color scales, including the color scales Aqua, Blackhot, Blaze, BlueRed, Gray, Hot, HSV, Iron, Red (Jet), Medical, Parula, Purple, Green (Rainbow) . 
To synchronize terahertz images with threedimensional video data in the Astrohn class, the method of advanced reading of terahertz video data is realized, the idea of which is as follows. The video data is read from the file and written to the temporary buffer. Each frame of video data is characterized by the time it was received from the device (in milliseconds, in a universal timeline beginning on January 1, 1970). As the buffer is full, data reading is suspended until the program accesses any data frame. You can access the data frame using the built-in predicate retrieve_timed_frame. This predicate checks if there is a frame in the buffer with a given timestamp $\mathrm{T}$, and, depending on the results of the check, performs the following actions:

If two frames with timestamps ti and $t i+1$ are present in the buffer, such that $\mathrm{ti} \leq \mathrm{T}$ and $\mathrm{T} \leq \mathrm{ti}$ +1 , then one of these frames is selected which is closest in time to T. The frame is transferred to the program for processing, in particular, the program receives an asynchronous message frame_obtained, signaling the receipt of the required frame of video data. Reading video data from a file is resumed and continues until the selected frame is in the middle of the buffer. As the buffer overflows, the oldest video frames are discarded.

If the last video frame in the buffer has a timestamp $\mathrm{tE}$, such that $\mathrm{tE}<\mathrm{T}$, reading of the video data into the buffer is resumed, and the execution of the predicate retrieve_timed_frame is suspended until a video frame with a suitable timestamp is received.

If the first time frame in the buffer has a timestamp $\mathrm{tB}$, such that $\mathrm{T}<\mathrm{tB}$, the execution of the predicate retrieve_timed_frame fails, the logical program rolls back.

The method of advanced reading of video data allows you to adaptively change the speed of reading a video file and synchronize the speed of reading video data received from different devices. The volume of three-dimensional video data obtained with a time-of-flight camera is much higher than the amount of terahertz data, so it is advisable to adapt the control of reading terahertz data, depending on the actually achieved rate of arrival of threedimensional video frames.

In Fig. 6 shows an example of a threedimensional image constructed by merging a point cloud obtained from a time-of-flight camera and a

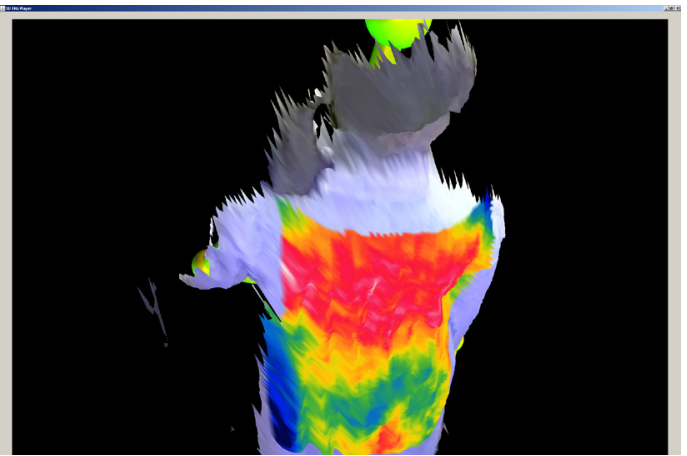

Fig. 6. An example of a terahertz video image projected on a three-dimensional surface of the human body. The terahertr image is placed against the visible. To display the terahert? data, the same color scale is used as in Fig. 1.

terahertz image. In Fig. 7, the same data is displayed, but the terahertz image is combined with the human skeleton calculated by the standard SDK of the Kinekt 2 device. Using a 3D match table allows you to project a terahertz image onto a three-dimensional surface at a real-time tempo. In particular, the threedimensional video shown in the figures can be rotated, scaled, or shifted with the mouse directly during playback.

\section{CONCLUSION}

As a basis for intelligent terahertz video surveillance, a method of semantic fusion of terahertz video with video images obtained on the basis of other physical principles, in particular, with a three-dimensional video image obtained with a time-of-flight camera, is proposed. For experiments with terahertz video surveillance, tools for object-oriented logical programming have been created, namely, builtin classes of the Actor Prolog language for input, recording, reading and synchronizing video streams

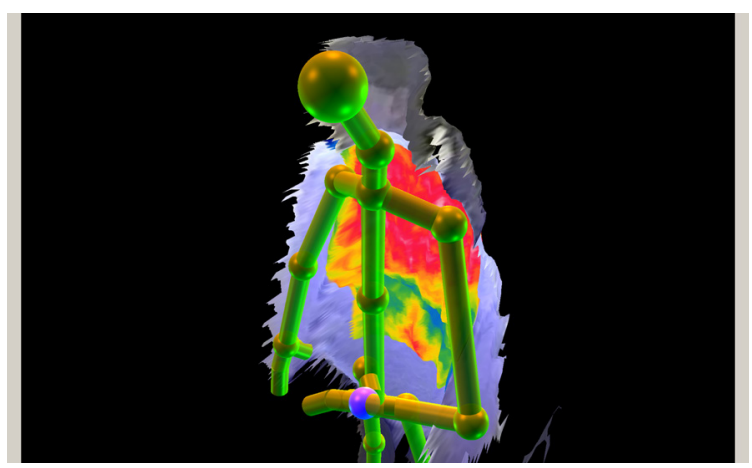

Fig. 7. An example of a terahertz video image projected on a three-dimensional surface of the human body. The three-dimensional surface is combined with the skeleton, which reflects the position of the head, arms, legs and other parts of the human body relative to the terahertr. video image. 
in terahertz and visible frequency ranges, and also three-dimensional video. The experimental verification demonstrated that the developed tools allow in real time to project a terahertz video image onto a three-dimensional surface of a person obtained with a time-of-flight camera. This information can be used by algorithms of automatic analysis to determine the position of a person and certain parts of his body with respect to a terahertz image, which will improve the quality of recognition of objects hidden under clothing. With the help of the developed tools, samples of multi-channel heterogeneous video images of people, weapons and objects were collected, which will later be used to teach algorithms for recognizing objects hidden under clothing.

\section{ACKNOLEDGMENT}

The authors are grateful to IA. Kershneru and R.A. Tolmacheva for participating in ongoing experiments, as well as companies Astron CJSC and ASoft Ltd. for providing equipment for recording teraherts, radiation THERZ-7A. The study was carried out with the support of the Russian Foundation for Basic Research (Project No. 16-29-09626-OI).

\section{REFERENCES}

1. Vollmer M, Möllmann KP. Infrared Thermal Imaging. Fundamentals, Research and Applications. Weinheim, WILEY-VCH, 2010, 612 p.

2. Chan WL, Deibel J, Mittleman DM. Imaging with terahertz radiation. Reports on progress in physics. 2007, 70(8):1325.

3. Sethy PK, Mishra PR, Behera S. An Introduction to Terahertz Technology, Its History, Properties and Application. Intern. conf. on computing and communication, 2015.

4. Zhu L, Suomalainen J, Liu J, Hyyppä J, Kaartinen H, Haggren H. A Review: Remote Sensing Sensors. In: Rustamov RB, Hasanova S, Zeynalova MH, editors. Multi-purposeful Application of Geospatial Data. IntechOpen, 2018, p. 19-42.

5. Sanders-Reed JN. Applications and challenges for MMW and THz sensors. In: Micro- and Nanotechnology Sensors, Systems, and Applications VII. Proc. of SPIE, 2015, vol. 9467, p. $94672 \mathrm{E}$.

6. Appleby R, Robertson DA, Wikner D. Millimeter wave imaging: a historical review. Proc. SPIE
Passive and Active Millimeter-Wave Imaging XX, 2017, vol. 10189, p. 1018902.

7. Sizov FF. Infrared and terahertz in biomedicine. Semiconductor Physics, Quantum Electronics \& Optoelectronics, 2017, 20(3):273-283.

8. Cherevko AG, Iljin EM, Morgachev JD, Polubehin AI. Analysis of patent activity in the field of advanced radar technology in extra-long infrared wavelength range. Vestnik SibGUTI, 2015, 2:164-173 (in Russ.).

9. Jackson JB, Bowen J, Walker G, Labaune J, Mourou $\mathrm{G}$, Menu $\mathrm{M}$, et al. A survey of terahertz applications in cultural heritage conservation science. IEEE Transactions on Terahertr. Science and Technology, 2011, 1(1):220-231.

10. Kemp MC. A review of millimetre-wave and terahertz technology for detection of concealed threats. Proc. IEEE 33rd Intern. Conf. on Infrared and Millimeter Waves, 2008, p. 1-2.

11. Kemp MC. Millimetre wave and terahertz technology for detection of concealed threats - a review. Proc. IEEE Joint 32nd Intern. Conf. on Infrared and Millimeter Waves, 2007, p. 647-648.

12. Taylor ZD, Singh RS, Bennett DB, Tewari P, Kealey CP, Bajwa N, et al. THz medical imaging: in vivo hydration sensing. IEEE transactions on terahertz science and technology, 2011, 1(1):201-219.

13. Antsiperov VE. Automatic target recognition algorithm for lowcount terahertz images. Computer Optics, 2016, 40(5):746-751.

14. Astahov SP, Stroev NN, Sulimski ES. Features of the Software for Design of Research Complex Using Subteraherz Matrix Camera. Mathematical morphology, 2015, 14(1) (in Russ.).

15. Kowalski M, Kastek M, Piszczek M, Życzkowski M, Szustakowski M. Harmless screening of humans for the detection of concealed objects. WIT Transactions on The Built Environment, 2015, 151:215-223.

16. Angeluts AA, Balakin AV, Borodin AV, Borodin AV, Esaulkov MN, Nazarov MM, et al. Terahertz Spectroscopy and Imaging. Russian Foundation for Basic Research Journal, 2014, 83(3):21-36 (in Russ.).

17. Ozhegov R, Gorshkov K, Vachtomin YB, Smirnov K, Finkel M, Goltsman G, etal. Terahertz imaging system based on superconducting heterodyne integrated receiver. In: $\mathrm{TH}_{z}$ and Security Applications. Springer; 2014, p. 113-125. 
18. Chrzanowski K. Testing passive surveillance terahertz imagers. Optica Applicata, 2013, 43(2):359-371.

19. Trofimov VA, Trofimov VV, Deng C, Zhao Y, Zhang C, Zhang X. Possible way for increasing the quality of imaging from $\mathrm{THz}$ passive device. Proc. of SPIE, 2011, Vol. 8189:81890L.

20. Cooper KB, Dengler RJ, Llombart N, Thomas B, Chattopadhyay G, Siegel PH. THz imaging radar for standoff personnel screening. IEEE Transactions on Terahertz. Science and Technology, 2011, 1(1):169-182.

21. Shen X, Dietlein CR, Grossman E, Popovic Z, Meyer FG. Detection and segmentation of concealed objects in terahertz images. IEEE Transactions on Image Processing, 2008, 17(12):2465-2475.

22. Semenov A, Richter H, Böttger U, Hübers HW. Imaging terahertz radar for security applications. Terabertz for Military and Security Applications VI, 2008, vol. 6949:694902.

23. Kemp MC. Detecting hidden objects: Security imaging using millimetre-waves and terahertz. IEEE Conference on Advanced Video and Signal Based Surveillance, 2007, p. 7-9.

24. Federici JF, Schulkin B, Huang F, Gary D, Barat $\mathrm{R}$, Oliveira F, et al. THz imaging and sensing for security applications - explosives, weapons and drugs. Semiconductor Science and Technology, 2005, 20(7):S266.

25. Spiegel W, Weg C, Henneberger R, Zimmermann $\mathrm{R}$, Roskos HG. Illumination aspects in active terahertz imaging. IEEE Trans. Microw. Theory Tech, 2010, 58(7):2008-2013.

26. Friederich F, Spiegel W, Bauer M, Meng F, Thomson MD, Boppel S, et al. THz active imaging systems with realtime capabilities. IEEE Transactions on Terahertz. Science and Technology, 2011, 1(1):183-200.

27. Pfeiffer UR, Zhao Y, Grzyb J, Al Hadi R, Sarmah $\mathrm{N}$, Förster $\mathrm{W}$, et al. A $0.53 \mathrm{THz}$ reconfigurable source module with up to $1 \mathrm{~mW}$ radiated power for diffuse illumination in terahertz imaging applications. IEEE Journal of Solid-State Circuits, 2014, 49(12):2938-2950.

28. Kowalski M, Piszczek M, Palka N, Szustakowski M. The methodology of THz-VIS fused images evaluation. Photonics Letters of Poland, 2013, 5(1):32-34.
29. Stroev NN, Sulimski ES. Research of means of multiframes representation for application in systems of radiovision of subterahertz range. Yugra State University Bulletin, 2016, (2):17-20 (in Russ.).

30. Trofimov VA, Trofimov VV, Shestakov IL, Blednov RG, Kovalev VY. Effective algorithm based on Fourier transform for the passive $\mathrm{THz}$ image quality enhancement. Image Sensing Technologies: Materials, Devices, Systems, and Applications IV, 2017, vol. 10209:1020907.

31. Trofimov VA, Trofimov VV. New algorithm for the passive $\mathrm{THz}$ image quality enhancement. Terahertr. Physics, Devices, and Systems X: Advanced Applications in Industry and Defense. 2016, vol. 9856:98560M.

32. Trofimov VA, Trofimov VV, Palka N, Kowalski M. Computer Processing of Images Captured with a Commercially Available $\mathrm{THz}$ Camera at Long Distances. Terahertz, and Mid Infrared Radiation: Detection of Explosives and CBRN (Using Terahertw). Springer, 2014, p. 167-174.

33. Trofimov VA, Trofimov VV, Kuchik IE, Zhang $\mathrm{Cl}$, Deng C, Zhao Ym, et al. Computer processing of image captured by the passive $\mathrm{THz}$ imaging device as an effective tool for its de-noising. Infrared, Millimeter-Wave, and Teraherts. Technologies II, 2012, vol. 8562:856207.

34. Cooper K, Dengler R, Llombart N, Bryllert T, Chattopadhyay G, Mehdi I, et al. An approach for sub-second imaging of concealed objects using terahertz $(\mathrm{THz})$ radar. Journal of Infrared, Millimeter, and Teraherts, Waves, 2009, 30(12):1297-1307.

35. Ramasamy Ramamurthy S, Roy N. Recent trends in machine learning for human activity recognition - A survey. Wiley Interdisciplinary Reviews: Data Mining and Knowledge Discovery, 2018, p. e1254.

36. Vineet V, Miksik O, Lidegaard M, Nießner M, Golodetz S, Prisacariu VA, et al. Incremental dense semantic stereo fusion for largescale semantic scene reconstruction. IEEE International Conference on Robotics and Automation, 2015, p. 75-82.

37. Oliveira L, Nunes U, Peixoto P, Silva M, Moita F. Semantic fusion of laser and vision in pedestrian detection. Pattern Recognition, 2010, 43:3648-3659. 
38. Lewis JJ, O'Callaghan RJ, Nikolov SG, Bull DR, Canagarajah N. Pixel-and region-based image fusion with complex wavelets. Information fusion, 2007, 8(2):119-130.

39. Mittleman DM. Twenty years of terahertz imaging. Optics express, 2018, 26(8):9417-9431.

40. Guillet JP, Recur B, Frederique L, Bousquet B, Canioni L, Manek-Hönninger I, et al. Review of terahertz tomography techniques. Journal of Infrared, Millimeter, and Terahertz. Waves, 2014, 35(4):382-411.

41. Zhang XC. Three-dimensional terahertz wave imaging. Philosophical Transactions of the Royal Society of London A: Mathematical, Physical and Engineering Sciences, 2004, 362(1815):283-299.

42. Gao J, Cui Z, Cheng B, Qin Y, Deng X, Deng B, et al. Fast Three-Dimensional Image Reconstruction of a Standoff Screening System in the Terahertz Regime. IEEE Transactions on Terahertz Science and Technology, 2018, 8(1):38-51.

43. Nowok S, Herschel R, Zimmermann R, Shoykhetbrod A, Lang SA, Pohl N. 3D Imaging System Based on a MIMO Approach at $360 \mathrm{GHz}$ for Security Screening. Proc. 37th PIERS: Progress In Electromagnetic Research Symposium, Shanghai, China; 2016, p. 671-675.

44. Zhou M. A Terahertz Holography Imaging System for Concealed Weapon Detection Application. School of Electronic and Engineering and Computer Science, Queen Mary, University of London, 2017 , p. 219.

45. Liu W, Li C, Sun Z, Zhang Q, Fang G. Threedimensional sparse image reconstruction for terahertz surface layer holography with random step frequency. Optics letters, 2015, 40(14):3384-3387.

46. Jun Takayanagi, Hiroki Jinno, Shingo Ichino et al. High-resolution time-of-flight terahertz tomography using a femtosecond fiber laser. Optics Express, 2009, 17(9):7533-7539

47. Hao J, Li J, Pi Y. Three-Dimensional Imaging of Terahertz Circular SAR with Sparse Linear Array. Sensors (Basel, Switzerland), 2018, 18(8):2477.

48. Rezgui ND, Andrews DA, Bowring NJ. Ultra Wide Band 3D Microwave Imaging Scanner for the Detection of Concealed Weapons. Proc. SPIE Millimetre Wave and Teraherts. Sensors and Technology VIII, 2015, vol. 9651:965108-1.
49. Gu S, Li C, Gao X, Sun Z, Fang G. Terahertz aperture synthesized imaging with fan-beam scanning for personnel screening. IEEE transactions on microwave theory and techniques, 2012, 60(12):3877-3885.

50. Chen S, Luo CG, Wang HQ, Peng L, Deng B, Zhuang ZW. Three-Dimensional Terahertz Coded-Aperture Imaging in Space Domain. IEEE Access, 2018, 6:32727-32736.

51. Chen S, Luo C, Wang H, Wang W, Peng L, Zhuang Z. Three-Dimensional Terahertz CodedAperture Imaging Based on Back Projection. Sensors (Basel, Switzerland), 2018, 18(8):2510.

52. Chen S, Luo C, Wang H, Deng B, Cheng Y, Zhuang Z. Three-Dimensional terahertz codedaperture imaging based on matched filtering and convolutional neural network. Sensors (Basel, Switzerland), 2018, 18(5):1342.

53. Chen S, Luo C, Deng B, Wang H, ID YC. Three-Dimensional Terahertz Coded-Aperture Imaging Based on Single Input Multiple Output Technology. Sensors, 2018, 18(1):303.

54. Chen S, Hua X, Wang H, Luo C, Cheng Y, Deng B. Three- Dimensional Terahertz Coded-Aperture Imaging Based on Geometric Measures. Sensors, 2018, 18(5):1582.

55. Trontelj J, Sešek A. Electronic terahertz imaging for security applications. Terahertz, RF, Millimeter, and Submillimeter-Wave Technology and Applications IX, 2016, vol. 9747:974713.

56. Gollub J, Yurduseven O, Trofatter K, Arnitz D, Imani M, Sleasman T, et al. Large metasurface aperture for millimeter wave computational imaging at the human-scale. Scientific reports, 2017, 7:42650.

57. Engström P, Axelsson M, Karlsson M. Microsoft Kinect for $\mathrm{THz}$ Sensor Management. Proc. 3rd Intern. Conf. on 3D Body Scanning Technologies, Lugano, Switzerland, Hometrica Consulting; 2012, p. 311-319.

58. Engström P, Axelsson M, Karlsson M. Combining structured light and Ladar for pose tracking in $\mathrm{THz}$ sensor management. Signal Processing, Sensor Fusion, and Target Recognition XXII, 2013, vol. 8745:874515.

59. Axelsson M, Karlsson M, Rudner S. A multicamera positioning system for steering of a $\mathrm{THz}$ stand-off scanner. Passive Millimeter-Wave Imaging Technology XIV, 2011, vol. 8022:80220L. 
60. Natour GE, Ait-Aider O, Rouveure R, Berry F, Faure P. Toward 3D reconstruction of Outdoor Scenes using an MMW radar and a monocular vision sensor. Sensors, 2015, 15(10):25937-25967.

61. Hantscher S, Essen H, Warok P, Zimmermann R, Schröder M, Sommer R, et al. LAOTSE, an Approach for Foreign Object Detection by multimodal netted 2D/3D Sensors. IEEE Tyrrhenian Intern. Workshop on Digital Communications-Enhanced Surveillance of Aircraft and Vehicles, 2011, p. 61-64.

62. Angeluts AA, Gapeyev AB, Esaulkov MN, Kosareva OG, Matyunin SN, Nazarov MM, et al. Study of terahertz-radiation-induced DNA damage in human blood leukocytes. Quantum Electronics, 2014, 44(3):247.

63. Rahman A, Rahman AK, Rao B. Early detection of skin cancer via terahertz spectral profiling and 3D imaging. Biosensors and Bioelectronics, 2016, 82:64-70.

64. Jeunesse P, Schmidhammer U. "On-the-Fly" Monitoring With a Single-Shot Terahertz TimeDomain Spectrometer. IEEE Sensors Journal, 2013, 13(1):44-49.

65. Baker C, Lo T, Tribe W, Cole B, Hogbin M, Kemp M. Detection of concealed explosives at a distance using terahertz technology. Proc. of the IEEE. 2007, 95(8):1559-1565.

66. Chernov G, Chernov V, Flores MB. 3D dynamic thermography system for biomedical applications. In: Application of Infrared to Biomedical Sciences. Springer; 2017, p. 517-545.

67. Rangel J, Soldan S, Kroll A. 3D thermal imaging: Fusion of thermography and depth cameras, robotics and autimation. IEEE Intern. Conf. on Quantitative InfraRed Thermography; 2014, pp.2311-2318.

68. Skala K, Lipić T, Sović I, Gjenero L, Grubišić I. 4D thermal imaging system for medical applications. Periodicum biologorum, 2011, 113(4):407-416.

69. Morozov AA, Sushkova OS, Petrova NG, Khokhlova MN, Migniot C. Development of Agent Logic Programming Means for Multichannel Intelligent Video Surveillance. Radioelektronika. Nanosistemy. Informacionnye Tebnologii (RENSIT), 2018, 10(1):101-116.

70. Bouguet JY. Camera Calibration Toolbox for Matlab. Pasadena, U.S., 2015. [Online] Available from: http:// www.vision.caltech.edu/bouguetj/calib_doc/htmls/ ref.html.

71. Morozov AA, Sushkova OS, Polupanov AF. Object-oriented logic programming of $3 \mathrm{D}$ intelligent video surveillance systems: The problem statement. RENSIT, 2017, 2(9):205-214.

72. Morozov AA, Sushkova OS. Real-time analysis of video by means of the Actor Prolog language. Computer Optics, 2016, 40(6):947-957.

73. Morozov AA, Sushkova OS, Polupanov AF. Towards the Distributed Logic Programming of Intelligent Visual Surveillance Applications. In: Pichardo-Lagunas O, Miranda-Jimenez S, editors. Advances in Soft Computing, Part II. Cham: Springer, 2017, p. 42-53.

74. Morozov AA. Development of a Method for Intelligent Video Monitoring of Abnormal Behavior of People Based on Parallel ObjectOriented Logic Programming. Pattern Recognition and Image Analysis, 2015, 25(3):481-492.

75. Morozov AA, Vaish A, Polupanov AF, Antciperov VE, Lychkov II, Alfimtsev AN, et al. Development of concurrent object-oriented logic programming platform for the intelligent monitoring of anomalous human activities. BIOSTEC 2014, vol. 511 of CCIS, Heidelberg, Springer, 2015, p. 82-97.

76. Morozov AA, Vaish A, Polupanov AF, Antciperov VE, Lychkov II, Alfimtsev AN, et al. Development of concurrent object-oriented logic programming system to intelligent monitoring of anomalous human activities. BIODEVICES 2014. SCITEPRESS, 2014, p. 53-62.

77. Morozov AA. Visual Logic Programming Based on the SADT diagrams. ICLP 2007, LNCS 4670, Heidelberg, Springer, 2007, p. 436-437.

78. Morozov AA. Development and Application of Logical Actors Mathematical Apparatus for Logic Programming of Web Agents. ICLP 2003, LNCS 2916, Heidelberg, Springer, 2003, p. 494-495.

79. Morozov AA. Logic Object-Oriented Model of Asynchronous Concurrent Computations. Pattern Recognition and Image Analysis, 2003, 13(4):640-649.

80. Morozov AA, Obukhov YV. An Approach to Logic Programming of Intelligent Agents for Searching and Recognizing Information on the 
Internet. Pattern Recognition and Image Analysis, 2001, 11(3):570-582.

81. Morozov AA. Actor Prolog: an Object-Oriented Language with the Classical Declarative Semantics. IDL-1999, Paris, France, 1999, p. 39-53.

82. Morozov AA. The Prolog with Actors. Programmirovanie, 1994, (5):66-78 (in Russ.).

83. Morozov AA, Sushkova OS. The Intelligent Visual Surveillance Logic Programming Web Site, 2018, Available from: http://www.fullvision.ru. 